\title{
FLEXIBLE ICA IN COMPLEX AND NONLINEAR ENVIRONMENT BY AN MUTUAL INFORMATION APPROACH
}

\author{
Daniele Vigliano, Michele Scarpiniti, Raffaele Parisi and Aurelio Uncini \\ INFOCOM Department, Università degli Studi di Roma “La Sapienza”, \\ Via Eudossiana 18, 00184, Roma, Italy \\ phone: + (39) 0683059551, fax: + (39) 0644585486, email: daniele.vigliano@poste.it
}

\begin{abstract}
ABASTRACT
This paper introduces an Independent Component Analysis (ICA) approach to the separation of nonlinear mixtures in the complex domain. Source separation is performed by the minimization of output mutual information (MMI approach). Nonlinear complex functions involved in the processing are realized by the so called "splitting functions" which work on the real and the imaginary part of the signal respectively. Some experimental results that demonstrate the effectiveness of the proposed method are shown.
\end{abstract}

\section{INTRODUCTION}

In the last years Blind Source Separation (BSS) realized through Independent Component Analysis (ICA) have raised great interest in the signal processing community. Recently the problem of source separation has been extended to the complex domain [2], [3] due to the need of frequency domain signal processing which is quite common in telecommunication [14] and biomedical applications [15], [15].

One of the most critical issues in ICA is the matching between the pdf of sources (usually unknown) and the algorithm's parameters. In order to improve the pdf matching for the learning algorithm, the so called Flexible ICA was recently introduced in (see [9]). The most attractive property of Flexible ICA is the faster and more accurate learning obtained by estimation of the parameters related to the pdf of signals. In this context, spline-based nonlinear functions seem to be particularly appealing as score functions. In fact splines can model a very large number of nonlinear functions and can be easily adapted by suitably varying their control points, with low computational burden.

Recent studies on ICA in the real domain showed that source separation can be effectively performed also in the case of convolutive nonlinear mixing environments (see [7], [8] for an overview) but in case of the complex domain only linear instantaneous mixtures have been considered until now [10], [11].

This paper extends the Post Nonlinear (PNL) mixture, well-known in literature in the case of the real domain, to the complex domain (complex-PNL). This extension requires proper modelling of the nonlinear distorting functions. here performed by use of the splitting functions already described in [6].
The paper is organized in the following way. Section II describes the complex domain mixing environment and analyzes the problem of separation. Section III introduces the demixing structure. Section IV presents the algorithm and the learning rules used to perform separation. Finally, section V describes some experimental results which show the effectiveness of the proposed approach.

\section{NONLINEAR COMPLEX MIXING MODEL}

Let us consider a vector $\mathbf{s}[n]=\left\{s_{1}[n], \ldots, s_{N}[n]\right\}^{T}$ of $N$ complex sources at time $n\left(\mathrm{~s}[n] \in \mathbb{C}^{N \times 1}\right)$; the $h^{\text {th }}$ source can be expressed as $s_{h}[n]=s_{R h}[n]+j s_{I h}[n]$. The goal of BSS realized by conventional ICA is to recover the complex signal $\mathbf{s}[n]$ from some observations of the complex mixture $\mathbf{x}[n]=\left\{x_{1}[n], \ldots, x_{N}[n]\right\}^{T}$. In this paper the post nonlinear mixing model is extended to the complex domain by assuming

$$
\mathbf{x}[n]=\mathbf{x}_{R}[n]+j \mathbf{x}_{I}[n]=\mathcal{F}\{\mathbf{s}\}=\mathbf{F}\{\mathbf{A s}[n]\}
$$

where the nonlinear function $\mathbf{F}\left\{\mathbf{v}_{R}[n]+j \mathbf{v}_{I}[n]\right\}=\mathbf{F}_{R}\left\{\mathbf{v}_{R}[n]\right\}+j \mathbf{F}_{I}\left\{\mathbf{v}_{I}[n]\right\} \quad$ is the model of the nonlinear distortion in the complex domain and $\mathbf{F}_{R}\left\{\mathbf{v}_{R}[n]\right\}=\left[g_{R 1}\left(v_{R 1}[n]\right), \ldots, g_{R N}\left(v_{R N}[n]\right)\right]^{T}$ and $\mathbf{F}_{I}\left\{\mathbf{v}_{R}[n]\right\}=\left[g_{I 1}\left(v_{I 1}[n]\right), \ldots, g_{I N}\left(v_{I N}[n]\right)\right]^{T} . \mathbf{A}$ is an $N \times N$ matrix such that $a_{i j} \in \mathbb{C}$.

The model of nonlinear complex functions considered in this paper is made of two separated functions, one for the real and one for the imaginary part of the signal. These models, called splitting functions [6], [11] are widely employed in signal processing because they provide a representation of nonlinear environments adequate to most of applications. In addition they allow a simple realization. The complex domain mixing environment (1), represented in Figure 1, can be rewritten in the following way:

$$
\begin{gathered}
\tilde{\mathbf{x}}[n]=\left[\begin{array}{l}
\mathbf{x}_{R}[n] \\
\mathbf{x}_{I}[n]
\end{array}\right]=\left[\begin{array}{c}
\mathbf{F}_{R}\left\{\mathbf{v}_{R}[n]\right\} \\
\mathbf{F}_{I}\left\{\mathbf{v}_{I}[n]\right\}
\end{array}\right] \\
\tilde{\mathbf{v}}[n]=\left[\begin{array}{c}
\mathbf{v}_{R}[n] \\
\mathbf{v}_{I}[n]
\end{array}\right]=\left[\begin{array}{cc}
\mathbf{A}_{R} & -\mathbf{A}_{I} \\
\mathbf{A}_{I} & \mathbf{A}_{R}
\end{array}\right]\left[\begin{array}{l}
\mathbf{s}_{R}[n] \\
\mathbf{s}_{I}[n]
\end{array}\right]=\tilde{\mathbf{A}} \tilde{\mathbf{s}}
\end{gathered}
$$


where $\mathbf{A}_{\mathrm{R}}$ and $\mathbf{A}_{\mathrm{I}}$ are the real and imaginary parts of the complex mixing matrix $\mathbf{A}=\mathbf{A}_{R}+j \mathbf{A}_{j}$.

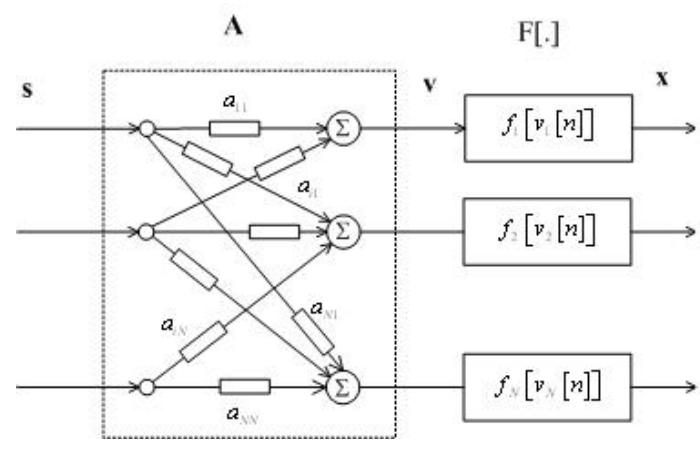

Figure 1. Complex mixing model.

Equations (2) with respect to the general model (1) have the very attractive property of involving only real quantities, thus making it possible to convert complex mixing models into real models of increased size.

\section{THE SEPARATION ISSUE AND NETWORK ARCHITECTURE}

One of the main issues in designing complex neural networks is the presence of complex nonlinear functions involved in the processing [10]-[12] i.e. complex score functions or distorting functions (for the nonlinear mixing environment). In literature are diffused several sets of properties which have to be satisfied by complex nonlinear functions in neural network applications. In particular Georgiou and Koutsougeras [13] defined the following five very interesting properties :
1. $f(z)=f(x, y)=u(x, y)+j v(x, y)$ nonlinear in $x$ and $y$
2. $f(z)$ is bounded: $|f(z)| \leq c<\infty$
3. $u_{x}, u_{y}, v_{x}, v_{y}$ exist and are bounded
4. $f(z)$ is not entire ${ }^{1}$
5. $u_{x} v_{y} \neq u_{y} v_{x}$

It should be noted that (3) requires the boundedness of the nonlinear function and its derivatives even when the function is defined in a local domain. By the Liouville theorem the cost for this restriction is that the function is not analytic.

According to the properties listed above, in order to overcome the dichotomy between boundedness and analyticity, complex nonlinear splitting functions have been introduced. In this approach real and imaginary parts are processed separately by real-valued nonlinear functions (see [14] and further [6] and [8] ).

The

$$
\text { splitting }
$$

functions

\footnotetext{
${ }^{1}$ A function $f(z)$ is said analytic in a point $z_{0}$ if its derivative exists throughout some neighborhood of $z_{0}$. If $f(z)$ is analytic in all points $z \in \mathbb{C}$, it is called entire.
}

$$
f(z)=f(x, y)=u(x)+j v(y)=f_{R}(\operatorname{Re}[z])+j f_{J}(\operatorname{Im}[z])
$$

avoid the problem of unboundedness of complex nonlinearities, as stated above, but they cannot be analytic.

It is now possible to define the demixing models and to design the network performing the source separation. As already mentioned, the linear mixing model is too unrealistic in a lot of practical situations but the issue of recovering the original sources in the more general non linear mixing model (1), with no particular a priori assumptions, is affected by a strong non-uniqueness [7], [8]. Several examples of this issue are widely diffused in literature.

In practice, the main issue becomes the research of theoretical conditions in terms of mixing environment and recovering architecture capable to guarantee the existence and the uniqueness of the solution.

In this paper an a priori knowledge on the mixing model was exploited to design the recovering network: the so called Mirror Model; this represents the architectural constraint which is sufficient to guarantee the existence and uniqueness of the solution (obviously up to some trivial indeterminacies). So on the basis of the knowledge of the mixing model it is possible to define the demixing model able to successfully perform the separation.

\section{Comment on independence}

Two complex random samples $y_{i}[n]$ and $y_{l}[n]$ of the output random vector $\mathbf{y}[n]$, are spatially independent if and only if $p_{\mathbf{y}}\left(y_{R, i}, y_{R, l}, y_{I, i}, y_{I, l}\right)=\prod_{h=i, l} p_{y_{R}}\left(y_{R, h}\right) p_{y_{I}}\left(y_{I, h}\right)$.

Considering this issue, two complex valued channels are independent not only if they are "separated" but if both the real and the imaginary part of the same signal are independent.

In any case the algorithm further introduced will be able to grant the source separation also when the real and imaginary parts are not strictly independent.

\subsection{The mirror model network architecture}

In detail, from the complex mixing model of (1) the complex mirror model is derived:

$$
\mathbf{y}[n]=\mathbf{y}_{R}[n]+j \mathbf{y}_{I}[n]=\mathbf{W} \cdot \mathbf{G}\{\mathbf{x}[n]\}
$$

In (4) $\mathbf{W}$ is an $N \times N$ complex matrix and $\mathbf{G}\left\{\mathbf{x}_{R}[n]+j \mathbf{x}_{I}[n]\right\}=\mathbf{G}_{R}\left\{\mathbf{x}_{R}[n]\right\}+j \mathbf{G}_{I}\left\{\mathbf{x}_{I}[n]\right\}$ models the nonlinear compensating functions in the complex domain. The nonlinear functions $\mathbf{G}\{\cdot\}$ have been realized as splitting functions according to (3). According to (2) it is possible to express the complex mixing model (4) by using real expressions only: 


$$
\tilde{\mathbf{y}}=\left[\begin{array}{l}
\mathbf{y}_{R}[n] \\
\mathbf{y}_{I}[n]
\end{array}\right]=\left[\begin{array}{cc}
\mathbf{W}_{R} & -\mathbf{W}_{I} \\
\mathbf{W}_{I} & \mathbf{W}_{R}
\end{array}\right]\left[\begin{array}{l}
\mathrm{G}_{R}\left\{\mathbf{x}_{R}[n]\right\} \\
\mathrm{G}_{I}\left\{\mathbf{x}_{I}[n]\right\}
\end{array}\right]=\tilde{\mathbf{W}} \cdot \tilde{\mathbf{G}}[\tilde{\mathbf{x}}]
$$

in which $\mathbf{G}_{R}\left\{\mathbf{x}_{R}[n]\right\}=\left[g_{R 1}\left(x_{R 1}\right), \cdots, g_{R N}\left(x_{R N}\right)\right]^{T}$ and $\mathbf{G}_{I}\left\{\mathbf{x}_{I}[n]\right\}=\left[g_{I 1}\left(x_{I 1}\right), \cdots, g_{I N}\left(x_{I N}\right)\right]^{T}$ are the real and imaginary part of the nonlinear compensating functions. Equation (5) represents a real-valued PNL demixing model and it preserves all the properties of demixing models in real domain.

Efficient design of the demixing strategy requires the choice of a proper demixing model, a cost function able to measure the independence of the outputs and an effective optimization method. In this section feedforward neural networks will be proposed and investigated as effective demixing models. Network parameters will be iteratively adapted (i.e. learned) on the basis of a measure of the output independence.

The architecture used to realize the model $\mathcal{F}\{\mathbf{G}, \mathbf{W}\}$ is represented in Figure 2. Nonlinear functions like the compensating function $g_{R i}\left(x_{R i}\right), g_{I i}\left(x_{I i}\right)$ or like the score functions $\psi_{R i}\left(y_{R i}\right), \psi_{I i}\left(y_{I i}\right)$ are realized by spline adaptive neurons (for more detail see [6], [10]). The closed form expression of spline neuron which represents the real and imaginary part of the $i^{\text {th }}$ channel is:

$$
\begin{aligned}
& g_{R i}\left(u_{R i}, \mathbf{Q}_{R i}\right)=1 / 2 \mathbf{T}_{R i}\left(u_{R i}\right) \cdot \mathbf{M} \cdot \mathbf{Q}_{R i} \\
& g_{I i}\left(u_{I i}, \mathbf{Q}_{I i}\right)=1 / 2 \mathbf{T}_{I i}\left(u_{I i}\right) \cdot \mathbf{M} \cdot \mathbf{Q}_{I i}
\end{aligned}
$$

where $\quad \mathbf{T}_{R i}\left(u_{R i}\right)=\left[\begin{array}{llll}u_{R i}{ }^{3} & u_{R i}{ }^{2} & u_{R i} & 1\end{array}\right] \quad$ and $\mathbf{T}_{I i}\left(u_{I i}\right)=\left[\begin{array}{llll}u_{I i}{ }^{3} & u_{I i}{ }^{2} & u_{I i} & 1\end{array}\right]\left(0 \leq u_{R}, u_{I}<1\right)$ and $\mathbf{M}$ is a matrix of coefficients which selects the properties of the interpolating curve. In this paper the Catmull-Rom spline has been used.

\section{ALGORITHM DERIVATION}

The demixing algorithm is based on output independence criterion [1]. This approach performs separation by minimization of the mutual information of the network output $\mathbf{y}$ (MMI approach). It uses an intuitively meaningful contrast function, it usually allows for simple learning rules and it is closely related to the other approaches based on information theory or high order statistic.

Considering a demixing model with parameters $\boldsymbol{\Phi}$, the cost function to be optimized is the mutual information of the output signals $\tilde{\mathbf{y}}$ :

$$
\begin{gathered}
\mathscr{L}\{\tilde{\mathbf{y}}[n], \boldsymbol{\Phi}\}=I(\tilde{\mathbf{y}})= \\
=E\left\{\log \left(p_{\tilde{\mathbf{x}}}(\tilde{\mathbf{x}})\right)\right\}-E\{\log (\operatorname{det}(\tilde{\mathbf{J}}))\}+ \\
-\sum_{i=1}^{N} E\left\{\log \left(p_{y_{R i}}\left(y_{R i}\right)\right)\right\}-\sum_{i=1}^{N} E\left\{\log \left(p_{y_{I i}}\left(y_{I i}\right)\right)\right\}
\end{gathered}
$$

In the output pdf $p_{\tilde{y}}(\tilde{\mathbf{y}})$ has been exploited to express the mutual information of $\tilde{\mathbf{y}}$ as a function of model's parameters and of $H(\tilde{\mathbf{x}})$ which does not depend on model's parameter:

$$
p_{\tilde{\mathbf{y}}}(\tilde{\mathbf{y}})=\frac{p_{\tilde{\mathbf{x}}}(\tilde{\mathbf{x}})}{\operatorname{det}(\tilde{\mathbf{J}})=}=\frac{p_{\tilde{\mathbf{x}}}(\tilde{\mathbf{x}})}{\operatorname{det}(\tilde{\mathbf{W}}) \cdot \prod_{k=1}^{N} \dot{g}_{R k} \prod_{k=1}^{N} \dot{g}_{l k}}
$$

In (10) $\tilde{\mathbf{J}}$ is the Jacobian matrix of the transformation between $\tilde{\mathbf{x}}$ and $\tilde{\mathbf{y}}$, while $g_{R i}$ and $g_{I i}$ are the generic elements of the real and imaginary part of nonlinear function $\mathbf{g}$.

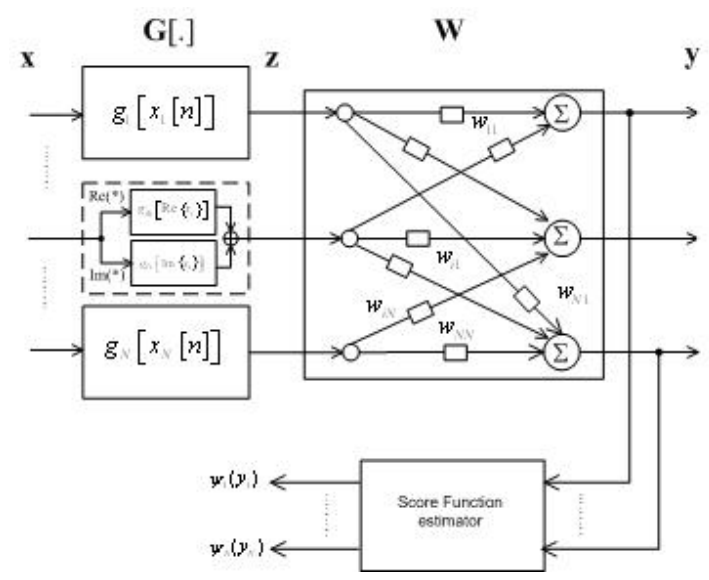

Figure 2. Demixing neural architecture.

The stochastic gradient and the natural gradient were used to minimize the with respect to the model parameter $\boldsymbol{\Phi}$, in particular the choice of gradient based procedure leads to consider the :

$$
\begin{aligned}
& \frac{\partial \log \left[p_{y_{R i}}\left(y_{R i}\right)\right]}{\partial \boldsymbol{\Phi}}=\frac{\dot{p}_{y_{R i}}\left(y_{R i}\right)}{p_{y_{R i}}\left(y_{R i}\right)} \frac{\partial y_{R i}}{\partial \boldsymbol{\Phi}}=\psi_{R i}\left(y_{R i}\right) \frac{\partial y_{R i}}{\partial \boldsymbol{\Phi}} \\
& \frac{\partial \log \left[p_{y_{I i}}\left(y_{I i}\right)\right]}{\partial \boldsymbol{\Phi}}=\frac{\dot{p}_{y_{I i}}\left(y_{I i}\right)}{p_{y_{R i}}\left(y_{I i}\right)} \frac{\partial y_{I i}}{\partial \boldsymbol{\Phi}}=\psi_{I i}\left(y_{I i}\right) \frac{\partial y_{I i}}{\partial \boldsymbol{\Phi}}
\end{aligned}
$$

where $\quad \psi_{R i}\left(y_{R i}\right)=\dot{p}_{R i}\left(y_{R i}\right) / p_{R i}\left(y_{R i}\right) \quad$ and $\psi_{I i}\left(y_{I i}\right)=\dot{p}_{I i}\left(y_{I i}\right) / p_{I i}\left(y_{I i}\right)$ are the so called score function (SF). Estimation of $\psi_{R i}\left(y_{R i}\right)$ and $\psi_{I i}\left(y_{I i}\right)$ are a critical step for the network learning.

In this work the conventional real-domain MMI algorithm have to be extended to the complex domain. This task can be accomplished by exploiting the splitting functions as attractive model of score functions [4], [6]. The most attractive property of the splitting score functions is the boundedness of real and imaginary part. Assuming

$$
\begin{aligned}
& \boldsymbol{\psi}=\boldsymbol{\psi}_{R}\left(\mathbf{y}_{R}\right)+j \boldsymbol{\psi}_{I}\left(\mathbf{y}_{I}\right) \\
& \psi_{i}=\psi_{R i}\left(y_{R i}\right)+j \psi_{I i}\left(y_{I i}\right)
\end{aligned}
$$


the expression of the complex vector $\boldsymbol{\Psi}$ can be rewritten (see (5) and (2)) by using only real terms:

$$
\tilde{\boldsymbol{\Psi}}=\left[\begin{array}{l}
\boldsymbol{\psi}_{R}\left\{\mathbf{y}_{R}[n]\right\} \\
\boldsymbol{\psi}_{I}\left\{\mathbf{y}_{I}[n]\right\}
\end{array}\right]
$$

In this way $\tilde{\boldsymbol{\Psi}}$ is a real vector of $2 N$ elements. Even if they are not strictly analytic, their profile is as smooth as required in the domain of existence. The learning of the score functions is performed in a direct way (see [1], [8]) minimizing the mean square error with respect to the spline's control points $\mathbf{Q}_{R}^{S C}$ and $\mathbf{Q}_{I}^{S C}$,

$$
\varepsilon_{j}=\frac{1}{2} E\left\{\left[\hat{\tilde{\psi}}_{j}\left(\tilde{y}_{j}, \boldsymbol{\Phi}\right)-\frac{\dot{p}_{\tilde{y}_{j}}\left(\tilde{y}_{j}\right)}{p_{\tilde{y}_{j}}\left(\tilde{y}_{j}\right)}\right]^{2}\right\}, j=1 \ldots N
$$

where $\tilde{\psi}_{j}$ is the spline model of the score function. The resulting rules are

$$
\begin{aligned}
\Delta \mathbf{Q}_{I}^{S C} & =\frac{1}{4} \mathbf{T}_{I} \mathbf{M} \mathbf{T}_{I} \mathbf{M} \mathbf{Q}_{I}^{S C}+\frac{1}{\Delta} \dot{\mathbf{T}}_{I} \mathbf{M} \\
\Delta \mathbf{Q}_{R}^{S C} & =\frac{1}{4} \mathbf{T}_{R} \mathbf{M} \mathbf{T}_{R} \mathbf{M} \mathbf{Q}_{R}^{S C}+\frac{1}{\Delta} \dot{\mathbf{T}}_{R} \mathbf{M}
\end{aligned}
$$

Having explored the mixing model, the associated cost function and the recovering network, the next step is to derive the learning rules for the others network's weights

$$
\begin{aligned}
& \Delta \mathbf{W}=\Delta \mathbf{W}_{R}+j \Delta \mathbf{W}_{I}=-\mathbf{W}^{-H}-\left[\psi_{R}+j \psi_{I}\right] \mathbf{y}^{H} \\
& \Delta \mathbf{Q}_{R, h, i+m}^{N L}=-\frac{\dot{\mathbf{T}}_{R h}(\mathbf{M})_{m}}{\dot{\mathbf{T}}_{R h} \mathbf{M} \mathbf{Q}_{R(\mathrm{G}), h, i+m}^{g_{R}}}+ \\
& -\sum_{k=1}^{N}\left(\psi_{R k} w_{R k n}+\psi_{I k} w_{I k n}\right) \frac{1}{2} \mathbf{T}_{R h}(\mathbf{M})_{m} \\
& \Delta \mathbf{Q}_{I, h, i+m}^{N L}=-\frac{\dot{\mathbf{T}}_{I h}(\mathbf{M})_{m}}{\dot{\mathbf{T}}_{I h} \mathbf{M} \mathbf{Q}_{I, h, i+m}^{g_{I}}}+ \\
& -\sum_{k=1}^{N}\left(\psi_{R k} w_{I k n}+\psi_{I k} w_{R k n}\right) \frac{1}{2} \mathbf{T}_{I h}(\mathbf{M})_{m}
\end{aligned}
$$

In these formulas $\psi_{R}=\left[\psi_{R 1}\left(y_{R 1}\right), \cdots \psi_{R N}\left(y_{R N}\right)\right]^{T}$, $\psi_{I}=\left[\psi_{I 1}\left(y_{I 1}\right), \cdots \quad \psi_{I N}\left(y_{I N}\right)\right]^{T}$ and $(\mathbf{M})_{m}$ is a vector composed by the $m^{\text {th }}$ column of the matrix $\mathbf{M}$.

\section{EXPERIMENTAL RESULT}

Several experimental tests were performed to assess the performance of the proposed architecture, with different number of sources, different mixing environments and different nonlinear distorting functions. This section describes the most significant results.

Signals were assumed to lie inside the range $[-1,1] \times[-1,1]$ and normalized so that each element of vector $\mathbf{v}$ in (see Figure 1) spans the range $[-0.8,0.8] \times[-0.8,0.8]$ (the complex signal is considered as a bidimensional signal).

For the test three complex signals with different distributions were considered: a 4-QAM signal, a uniform random signal and a PSK signal. The diversity in source distribution is properly faced by the flexibility of spline splitting activation function. The mixing environment (eq. (1)) is:

$$
\begin{gathered}
\mathbf{A}=\left[\begin{array}{ccc}
1.125-j 0.375 & -0.3-j 0.3 & -0.15+j 0.45 \\
-0.375+j 0.3 & 1.05+j 0.675 & -0.075+j 0.3 \\
0.15-j 0.3 & -0.45+j 0.6 & 0.975+j 0.45
\end{array}\right] \\
\mathbf{F}[\mathbf{v}]=\left[\begin{array}{c}
v_{R 1}+0.7 v_{R 1}^{3}+j\left(v_{I 1}+0.7 v_{I 1}^{3}\right) \\
v_{R 2}+0.7 \tanh \left(3 v_{R 2}\right)+j\left(v_{I 2}+0.7 \tanh \left(3 v_{I 2}\right)\right) \\
v_{R 3}+0.7 \tanh \left(3 v_{R 3}\right)+j\left(v_{I 3}+0.7 v_{I 3}^{3}\right)
\end{array}\right]
\end{gathered}
$$

The spline neurons of the recovering structure had 31 control points for the score functions and 21 for the nonlinear compensating functions. The learning rate were $\mu_{W}=5 * 10^{-3}, \mu_{\text {Spline }}=5 * 10^{-4}$. The training was stopped after 600 epochs.

The effectiveness of the separation is evidenced in Figure 3 that shows the joint pdf of the original sources (Figure $3 \mathrm{a}-\mathrm{c}$ ), of the nonlinear mixture (Figure $3 \mathrm{~d}-\mathrm{f}$ ) and finally of the separated signals (Figure $3 \mathrm{~g}-\mathrm{i}$ ).

In order to provide a mathematical evaluation of the output separation, different indexes of performance are available in literature. In this paper was adopted the separation index $S_{j}$ of the $j^{\text {th }}$ source [5], the closed form expression for the index is:

$$
S_{j}=10 \log \left[E\left\{\left(|y|_{\sigma(j), j}\right)^{2}\right\} / E\left\{\sum_{k \neq j}\left(|y|_{\sigma(j), k}\right)^{2}\right\}\right]
$$

In (15) $y_{i, j}$ is the $i^{\text {th }}$ output signal when only the $j^{\text {th }}$ input signal is present, while $\sigma(j)$ is the output channel corresponding to the $j^{\text {th }}$ input.

Figure 4 shows that after about 300 epochs the training is stable. So the profiles of the separation index $S_{j}$ for each channel assures the effectiveness of the learning.

Figure 5 shows the spline neurons which perform the nonlinear compensation for each channel; according to the model of splitting function each channel has a two compensating function for real and imaginary part.

\section{CONCLUSION}

In this paper a novel complex model of mixing environment has been introduced and described: the post nonlinear complex mixing model.

The BSS problem in this new environment is solved by exploiting an ICA-based algorithm. In particular, the proposed approach extends to complex domain the well-known MMI algorithm; it is based on the use of flexible spline networks to perform local on-line estimation of the score functions and nonlinear 
compensating functions. The extension to the complex domain is realized by exploiting the attractive properties of splitting functions.

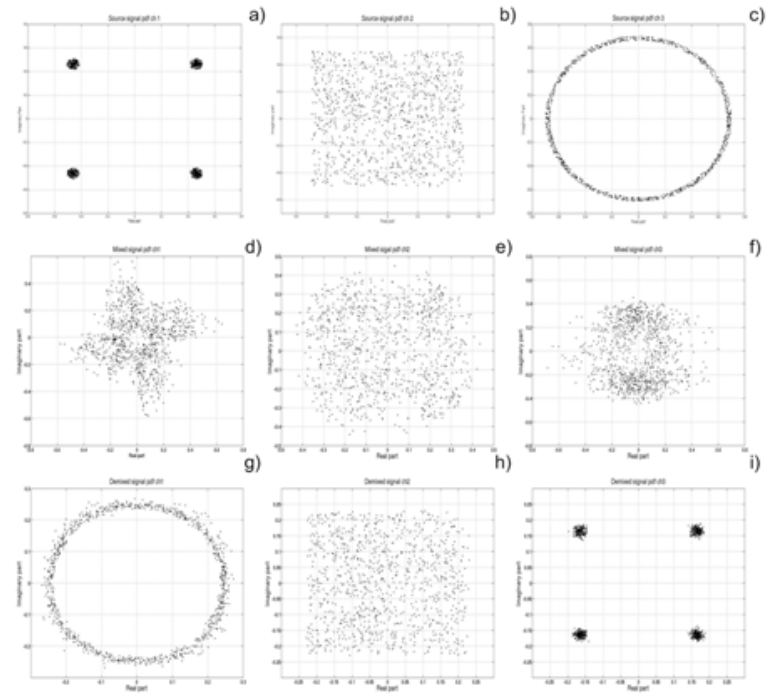

Figure 3. Joint pdf of the signal involved in separation; a)-c) original sources,d)-f) nonlinear mixture, g)-i) recovered sources.

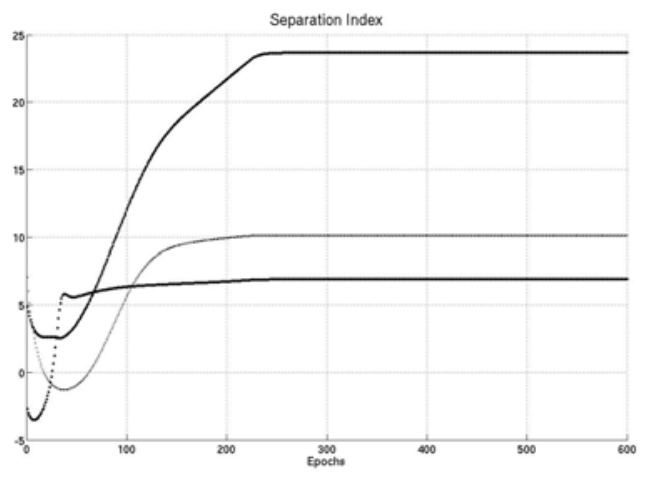

Figure 4 Separation index during training.
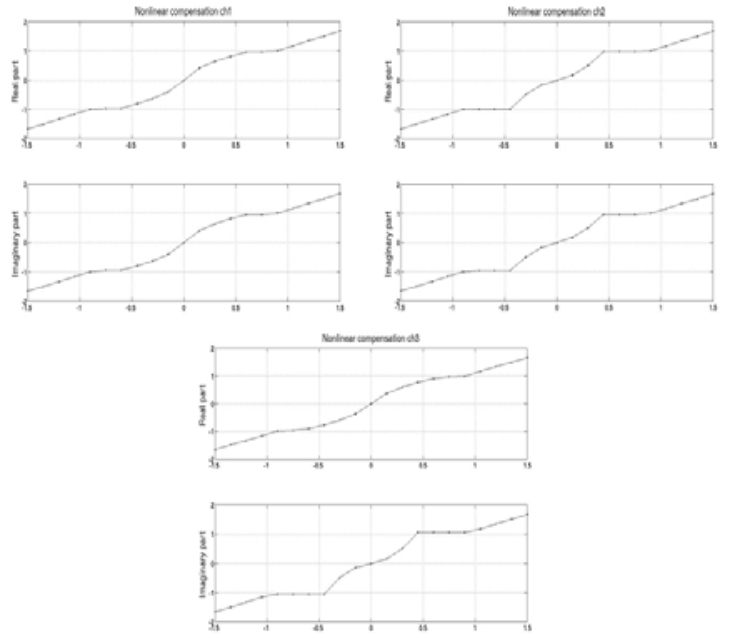

Figure 5 Splitting model of nonlinear compensating function realized by spline neurons.
Several tests have been performed to verify the effectiveness of the proposed approach; the quality of the separation has been evaluated in terms of the Separation Index which is widely diffused in literature.

\section{REFERENCES}

[1] Taleb and C. Jutten, "Source separation in post nonlinear mixtures", IEEE Trans. on Signal Processing, Vol. 47, 10, pp. 2807-2820, 1999.

[2] E. Bingham and A. Hyvärinen: "A fast fixed-point algorithm for independent component analysis of complex valued signals", International Journal of Neural Systems, Vol. 10, No. 1, February, 2000.

[3] S. Fiori, A. Uncini, F. Piazza, "Neural Blind Separation of Complex Sources by Extended APEX Algorithm (EAPEX)”, Proc. of IEEE ISCAS'99, Orlando (USA), vol. V, pp. 627-630, June 1999.

[4] P. Smaragdis, "Blind separation of convolved mixtures in the frequency domain" in Proc. International workshop on Indipendence \& Artificial Neural Networks, Tenerife, Spain, February 9-10 1998.

[5] D. Shobben, K. Torkkola, P. Smaragdis, "Evaluation of blind signal separation methods". In Proc. of ICA and BSS, Aussois, France, January 11-15, 1999.

[6] Uncini, F. Piazza, "Blind Signal Processing by Complex domain Adaptive Spline Neural Network”. In Transaction on Neural Networks, vol.14, no.2, Mar. 2003.

[7] C. Jutten, J. Karhunen, "Advances in Nonlinear Blind Sources Separation". 4th International Symposium on Independent Component Analysis and Blind Signal Separation (ICA2003), Nara, Japan, April 2003.

[8] D. Vigliano, R. Parisi, A. Uncini, “An Information Theoretic Approach to a Novel Nonlinear Independent Component Analysis Paradigm”, In Elsevier Information Theoretic Signal Processing, 85 Special Issue, pp. 997-1028, 2005.

[9] S. Fiori, "Blind Signal Processing by the Adaptive Activation function Neuron”. In Neural Networks, Vol. 13, No. 6, pp. 597-611, August 2000.

[10] T. Adali, T. Kim, and V. D. Calhoun, "Independent Component Analysis, by Complex Nonlinearities", in Proc. ICASSP 2004, vol.5, pp. 525-528, Montreal, Canada, 2004.

[11] V. D. Calhoun and T. Adali, "Complex Infomax: Convergence and Approximation of Infomax With Complex Nonlinearities", in Proc. NNSP, Switzerland, 2002.

[12] T. Kim, T. Adali, "Universal approximation of fully complex feed-forward neural network", in Proc. Of IEEE ICASSP'02, Vol. 1, pp. .973-976, Orlando, FL, 2002.

[13] G. M. Georgiou, C. Koutsougeras, “Complex Domain Backpropagation”, IEEE Trans. On Circuit and System II, Vol. 39, No. 5, pp. 330-334, May 1992.

[14] N. Benvenuto, M. Marchesi, F. Piazza, A. Uncini, "Nonlinear satellite radio links equalized using Blind Neural Network”, Proc. of ICASSP, Vol.3, pp.1521-1524, 1991.

[15] V. D. Calhoun, T. Adali, G. D. Pearlson, P. C. van Zijl, and J. J. Pekar, "Independent Component Analysis of FMRI Data in the Complex Domain”, Magn Reson. Med., vol. 48, pp. 180-192, 2002.

[16] V. D. Calhoun, T. Adali, G. D. Pearlson, and J. J. Pekar, "On Complex Infomax Applied to Complex FMRI Data”, in Proc. ICASSP, Orlando, FL, 2002. 